Bull. Mater. Sci., Vol. 36, No. 6, November 2013, pp. 1091-1095. (C) Indian Academy of Sciences.

\title{
Facile preparation of superhydrophobic surface with high adhesive forces based carbon/silica composite films
}

\author{
RUANBING HU ${ }^{\mathrm{a}, \mathrm{b}}$, GUOHUA JIANG ${ }^{\mathrm{a}, \mathrm{b}, *}$, XIAOHONG WANG ${ }^{\mathrm{a}, \mathrm{b}}$, XIAOGUANG XI ${ }^{\mathrm{a}, \mathrm{b}}$ \\ and RIJING WANG ${ }^{\mathrm{a}, \mathrm{b}}$ \\ ${ }^{a}$ Key Laboratory of Advanced Textile Materials and Manufacturing Technology (ATMT), Ministry of Education, \\ Zhejiang Sci-Tech University, Hangzhou 310018, People's Republic of China \\ ${ }^{\mathrm{b}}$ Department of Materials Engineering, College of Materials and Textile, Zhejiang Sci-Tech University, Hangzhou 310018, \\ People's Republic of China
}

MS received 23 May 2012; revised 14 July 2012

\begin{abstract}
Glass substrates modified by carbon/silica composites are fabricated through a two-step process for the preparation of a superhydrophobic surface (water contact angle $\geq 150^{\circ}$ ). Carbon nanoparticles were first prepared through a deposition process on glass using a hydrothermal synthesis route, then the glass was modified by $\mathrm{SiO}_{2}$ using the hydrolysis reaction of tetraethylorthosilicate at room temperature. It is not only a facile method to create a superhydrophobic surface, but also helps to form a multi-functional surface with high adhesive forces.
\end{abstract}

Keywords. Superhydrophobic surface; high adhesive forces; hydrothermal synthesis route; hydrolysis reaction.

\section{Introduction}

In recent years, bionics has attracted considerable attention for its great prospect. Shining drops of rain water forming a nearly perfect spherical shape on lotus leaves, which can be rolled off easily, is one of the most beautiful wonders of nature. This natural phenomenon is known as lotus effect (Zhai et al 2004; Cha et al 2010; Hipp et al 2010) and is considered as the basic study of superhydrophobic behaviour on a surface. Now the study of superhydrophobic behaviour of various surfaces is not only part of fundamental research, but also used in practical applications in many fields, especially in daily life (Yang et al 2007; Zhang et al 2011; Ogihara et al 2012), industry (Li et al 2008; Xu et al 2011) and agriculture (Sheng and Zhang 2009; Wang et al 2011). Cassie and Baxter (1944) as well as Wenzel (1936) proposed two mathematical models to explain the wetting phenomena on rough surfaces that leads to superhydrophobic property (Erbil and Cansoy 2009; Su et al 2009). Usually, any surface with water contact angle larger than $150^{\circ}$ can be defined as a superhydrophobic surface (Tuberquia et al 2010). Various materials and methods were tried out for the fabrication of superhydrophobic surfaces. Generally, these approaches to obtain superhydrophobicity can be divided into two groups, one of them is to achieve superhydrophobicity by changing the structure and surface energy of substrates. For example, Meng et al (2008) created superamphiphobic surfaces on common engineering metals by immersing them into perfluorocarboxylic acid and keeping for a few hours. Ishizaki and Sakamoto (2011) prepared colour-tuned superhydrophobic magnesium

\footnotetext{
*Author for correspondence (polymer_jiang@ hotmail.com)
}

alloy with corrosion resistance. Xiu et al (2008) obtained superhydrophobic surfaces on silicon surfaces by hierarchical etching. Shen et al (2010) employed a flower-like silicon particulate film by chemical etching and obtained superhydrophobicity. Another method is by coating superhydrophobic surface on the substrates such as Tsai and Lee (2007) who employed a raspberry-like particulate film prepared by layer-by-layer deposition and produced superhydrophobic surfaces. Yildirim et al (2011) prepared a superhydrophobic surface by coating fluorinated mesoporous silica nanoparticles. Brassard et al (2011) prepared superhydrophobic thin films by synthesizing monodisperse fluorinated silica nanoparticles. Manca et al (2009) obtained superhydrophobic surfaces by trimethylsilanized silica nanoparticles based sol-gel processing.

Recently, various methods were used to obtain superhydrophobic surfaces, such as template method, spray painting, self-assemble way and etching method. However, most of them employed just only one material to create a superhydrophobic surfaces, while few employed composites to create superhydrophobic surfaces. Here, we report the design of superhydrophobic surface using high adhesive forces by means of employing $\mathrm{C} / \mathrm{SiO}_{2}$ composites, which is simple and environmental friendly. Especially, the high adhesive forces enhance water-retaining capacity of the surface which is very important for long-distance transport of water. C nanoparticles were prepared through a deposition process on the glass using a hydrothermal synthesis route, then tetraethylorthosilicate (TEOS) dissolved in isopropanol (IPA) forming homogeneous solution, was dropped onto the glass modified by $\mathrm{C}$ nanostructures and kept in a container for a few days. $\mathrm{C} / \mathrm{SiO}_{2}$ composites were fabricated during the hydrolysis reaction of TEOS. In order to reduce 
surface energy, the glass was further treated with fluorosilicone. After that, the fabricated coatings exhibited superhydrophobic property because of their dual-size rough surface and low surface energy.

\section{Experimental}

\subsection{Materials}

All chemicals were of analytical grade and used as received. Glucose was purchased from Tianjin Henxing Chemical Reagent Manufacturing Co., Ltd. TEOS was obtained from Tianjin Kermal Chemical Reagent Co. Ltd. (IPA) was purchased from Hangzhou Gaojing Fine Chemicals Co. Ltd. 1H,1H,2H,2H-Perfluordecyltrimethoxysilane (>97 wt $\%$ ) was obtained from Nanjing Daoning Chemicals Co. Ltd.

\subsection{Methods}

A quantity of $6 \mathrm{~g}$ of glucose was added to $60 \mathrm{~mL}$ of deionized water by stirring for a few minutes, forming homogeneous solution. The resulting mixture was transferred to and sealed in a Teflon-lined autoclave, and a piece of glass was also inserted into the autoclave. The autoclave was sealed and maintained at $160{ }^{\circ} \mathrm{C}$ for $6 \mathrm{~h}$. After the autoclave was cooled down to room temperature naturally, the glass was collected and washed with deionized water carefully, and dried for $12 \mathrm{~h}$. Then TEOS was dissolved in IPA with different volume ratios forming homogeneous solution, which was dropped onto the glass modified by $\mathrm{C}$ nanostructures and kept in a container for a few days. The $\mathrm{C} / \mathrm{SiO}_{2}$ composites were fabricated during the hydrolysis reaction of TEOS, followed by perfluorosilane deposition to obtain high water-repellance.

\subsection{Characterization}

Microstructures of $\mathrm{SiO}_{2}$ and $\mathrm{C} / \mathrm{SiO}_{2}$ composites were investigated by ULTRA-55 field-emission scanning electron microscopy (FE-SEM) and the Fourier transform infrared (FT-IR) spectra were recorded on a Nicolet 5700 spectrophotometer. The wettability of the as-prepared $\mathrm{C} / \mathrm{SiO}_{2}$ composites films was analysed by measurement of the water angles using SL200B contact angle system at ambient temperature. Water droplets were dropped carefully onto the samples. The average contact angle was obtained by measuring at three different positions of the same sample, the accuracy of measurement was $\pm 1^{\circ}$.

\section{Results and discussion}

Figure 1 shows FE-SEM images of the resulting surface after the two-step process. In contrast to network-like $\mathrm{SiO}_{2}$ nanostructures which have single particle chains (figure $1 \mathrm{~b}$ ), the network-like $\mathrm{C} / \mathrm{SiO}_{2}$ composites (figure 1a) appear more rough.

FT-IR analyses were carried out on thin films deposited on the glass to provide a description of silica layer covering the carbon particles. The spectrum of $\mathrm{C}$ nanoparticles in figure 2(c) shows a broad band with a maximum breadth of around $3365 \mathrm{~cm}^{-1}$, which is attributed to the $\mathrm{O}-\mathrm{H}$ stretching. A small peak appearing around $2925 \mathrm{~cm}^{-1}$ is attributed to the stretching vibration of $\mathrm{C}-\mathrm{H}$ bond in the form of $\mathrm{CH}_{2}$ (Wan et al 2008). The stretching at $1282 \mathrm{~cm}^{-1}$ is attributed to the $\mathrm{C}-\mathrm{O}-\mathrm{C}$ skeleton. The spectrum of $\mathrm{C} / \mathrm{SiO}_{2}$ composites in figure 2(b) shows a broad band with a maximum breadth of around $3425 \mathrm{~cm}^{-1}$, which is attributed to the $\mathrm{O}-\mathrm{H}$ stretching. A strong peak appears at $1100 \mathrm{~cm}^{-1}$ due to the presence of $\mathrm{Si}-\mathrm{O}-\mathrm{Si}$ asymmetric bond stretching. Two small peaks appearing at $950 \mathrm{~cm}^{-1}$ and $2926 \mathrm{~cm}^{-1}$ are attributed to the stretching vibration of $\mathrm{Si}-\mathrm{OH}$ and $\mathrm{C}-\mathrm{H}$ bonds, respectively. The peak at $2700 \mathrm{~cm}^{-1}$ is attributed to the $\mathrm{C}-\mathrm{H}$ stretching vibration. The spectrum of fluorinated $\mathrm{C} / \mathrm{SiO}_{2}$ composites in figure 2(a) shows a small peak appearing at $960 \mathrm{~cm}^{-1}$, which is attributed to the stretching vibration of the $\mathrm{C}-\mathrm{F}$ bond in the form of $\mathrm{CF}_{3}$ (Brassard et al 2011).
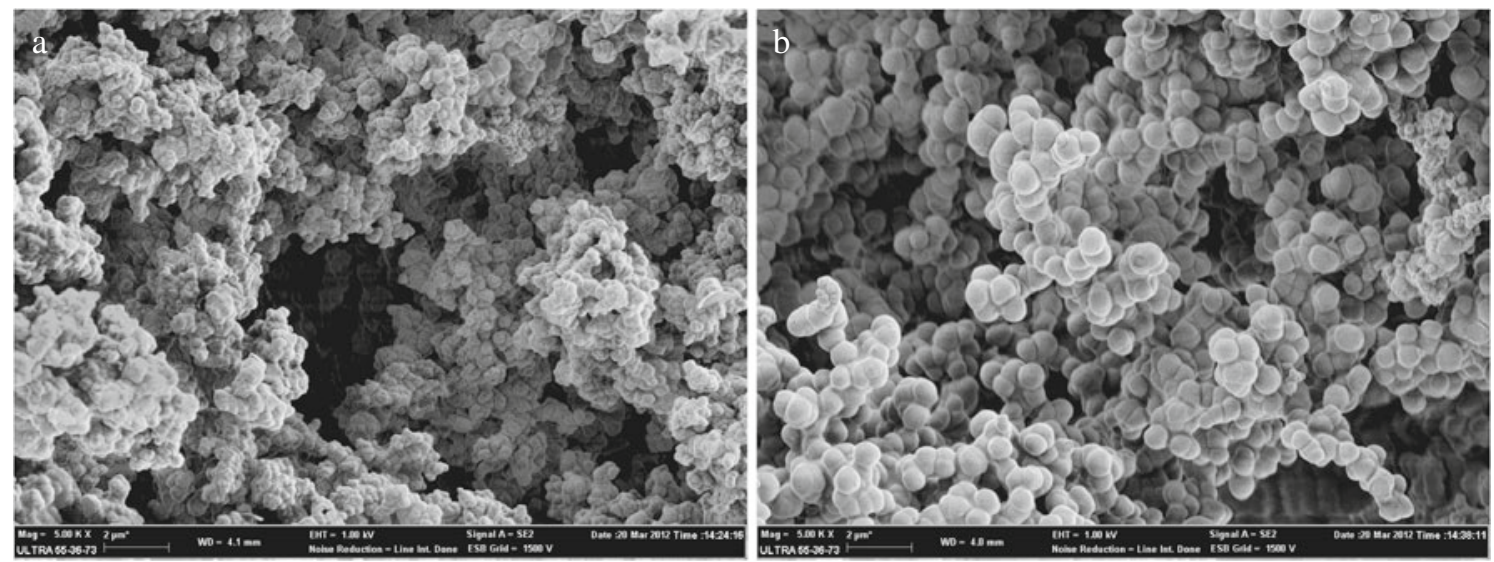

Figure 1. FE-SEM images of resulting surface after different processes: glass modified by (a) $\mathrm{C} / \mathrm{SiO}_{2}$ composites and (b) $\mathrm{SiO}_{2}$. All scale bars are of $2 \mu \mathrm{m}$. 
Water contact angle tests are carried out on thin films to obtain better understanding of wettability. $5 \mu \mathrm{L}$ water is dropped on the glass modified by $\mathrm{SiO}_{2}$, which is due to the presence of hydrophilic $\mathrm{Si}-\mathrm{OH}$ bonds and exhibits

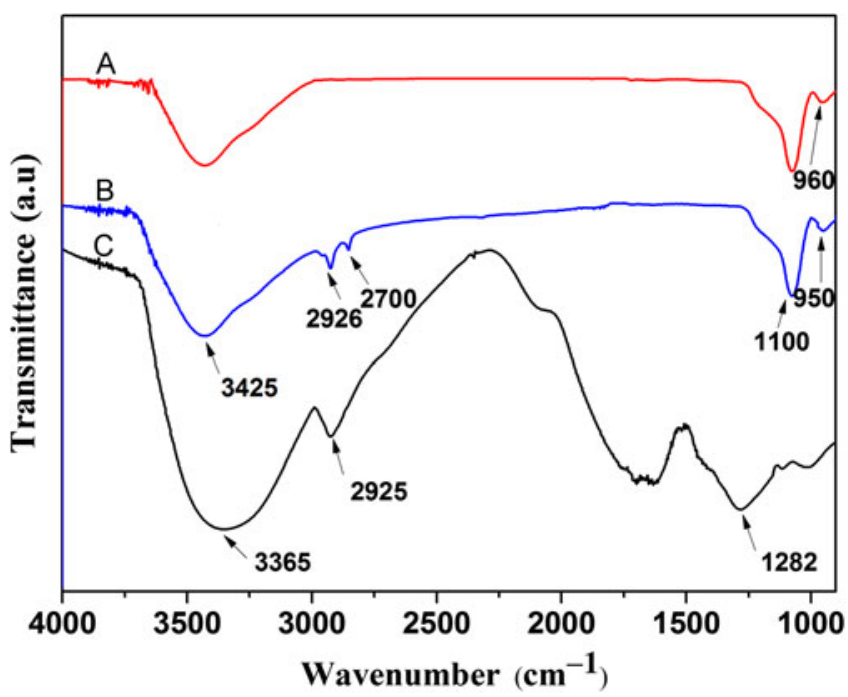

Figure 2. FT-IR spectra of thin films prepared by (a) fluorinated $\mathrm{C} / \mathrm{SiO}_{2}$ composites, (b) $\mathrm{C} / \mathrm{SiO}_{2}$ composites and (c) $\mathrm{C}$ nanoparticles. hydrophilic property. After treatment with fluorosilicone, its water contact angle (WCA) reaches $133^{\circ}$ (the inset of figure 3(a)). The wettability of glass modified by $\mathrm{C}$ nanoparticles is similar to that of glass modified by $\mathrm{SiO}_{2}$. However, when the glass is modified by $\mathrm{C} / \mathrm{SiO}_{2}$ composites, followed by treatment with fluorosilicone, its WCA is $152^{\circ}$ (inset of figure 3(b)), which indicates that the surface of the glass has superhydrophobic property. In addition, as IPA keeps increasing, WCA increases first, and then decreases gradually. The possible reason for this phenomenon is the changing of surface roughness at different volume ratios of TEOS and IPA. As shown in figure 3, when the volume ratios of IPA and TEOS are $2: 1$ or 5:1, the surface exhibits superhydrophobic properties.

Furthermore, we also find that when the drop is turned upside-down, it still adheres to the surface. As shown in figure 4 , the $\mathrm{C} / \mathrm{SiO}_{2}$ composite film modified by TEOS and IPA with volume ratio of 1:5 has strong adhesive force that is similar to a gecko's foot (Zeng et al 2009; Liu et al 2010; Bhushan 2012). A gecko's feet possess superhydrophobicity and high adhesive ability towards water, because they are covered with millions of well-aligned microscopic keratinous hairs called setae (20-70 $\mathrm{mm}$ in length and 3-7 mm in diameter), which are further split into hundreds of smaller nanoscale ends called spatulae (100-200 nm in diameter)
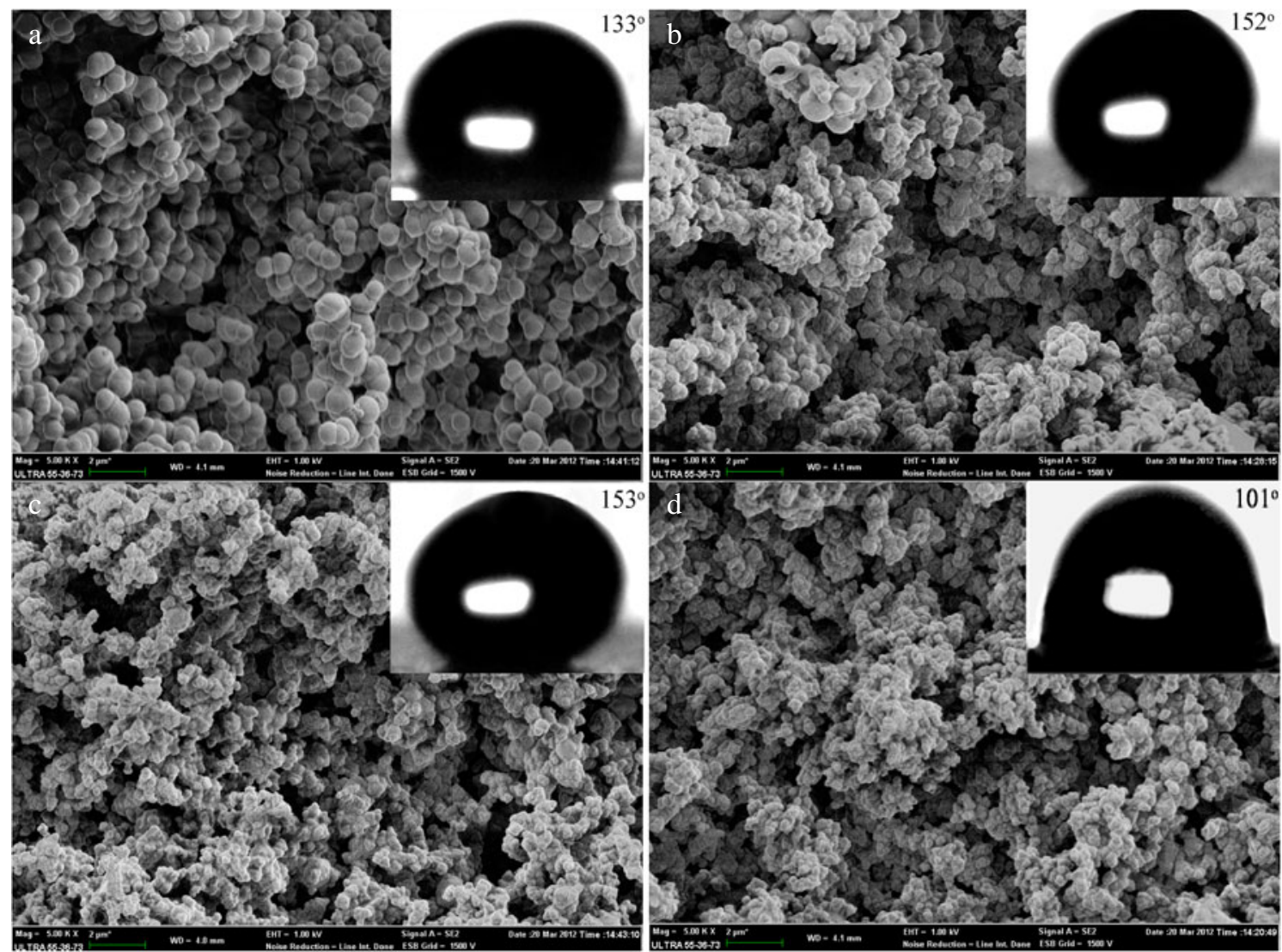

Figure 3. FE-SEM images of glasses modified by $\mathrm{C} / \mathrm{SiO}_{2}$ composites with different volume ratios of IPA and TEOS: 0 (a), 2 (b), 5 (c) and $10(\mathbf{d})$; insets are WCA images. All scale bars are of $2 \mu \mathrm{m}$. 


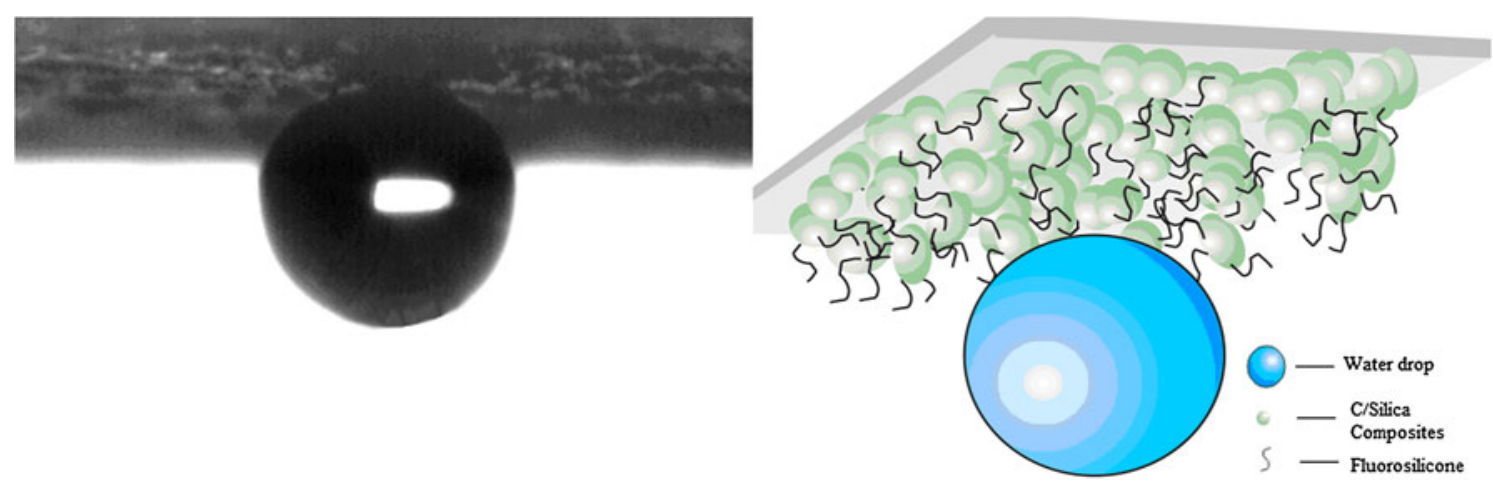

Figure 4. A 5- $\mu \mathrm{L}$ water drop hanging on micro-fluctuant area that is upside-down; left is WCA image, right is schematic of a $5-\mu \mathrm{L}$ water drop hanging on rough surface.

(Liu et al 2012). The possible reason for superhydrophobic surface with high adhesive force has been studied by many researchers such as $\mathrm{Li}$ and Shen (2011), Lai et al (2009) and Hong et al (2007). The carbon/silica composite films reported in this paper also exhibit strong adhesive forces. We suggest that the strong adhesion can be attributed to van der Waals force and negative pressure generated by the air trapped in a closed 'liquid-solid' system when the drop is turned upside-down. The micro-fluctuant surface and hair-like fluorosilicone result in a large contact area when a droplet is placed on it, thereby increasing the van der Waals force between the water droplet and surface. When the droplet is turned upside-down, the direction of gravity changes which leads to change in the surface energy of 'liquid-solid' and 'liquid-air' systems. Generally, the variation of surface energy results in negative pressure which is an important factor for the water droplet to adhere to the surface.

\section{Conclusions}

In summary, we designed a two-step process for the preparation of a superhydrophobic surface. A binary structure results from the combination of $\mathrm{C}$ nanoparticles and network-like $\mathrm{SiO}_{2}$ nanostructures, after treatment with fluorosilicone. The obtained surface has two functional areas that present different wettabilities and high adhesive force that enhances the water-retaining capacity of the surface. This feature can be used in places where water has to be retained for a long time. The advantages of this methodology also include its simplicity and ease of control.

\section{Acknowledgements}

This work was financially supported by the Qianjiang Talents Project of Zhejiang Province (2010R10023), Scientific Research Foundation for the Returned Overseas Chinese Scholars, State Education Ministry (1001603-C), Natural Science Foundation of Zhejiang Province (Y4100045), Training Foundation for the Excellent Young Talents by the Key Laboratory of Advanced Textile
Materials and Manufacturing Technology (ATMT), Ministry of Education (2011QN04) and the National Natural Science Foundation of China (51133006).

\section{References}

Bhushan B 2012 Langmuir 281698

Brassard J-D, Sarkar D K and Perron D 2011 ACS Appl. Mater. Interf. 33583

Cassie A B D and Baxter S 1944 Trans. Faraday Soc. 40546

Cha T-G, Yi J W, Moon M-W, Lee K-R and Kim H-Y 2010 Langmuir 268319

Erbil H-Y and Cansoy C-E 2009 Langmuir 2514135

Hipp B, Kunert I and Dürr M 2010 Langmuir 266557

Hong X, Gao X-F and Jiang L 2007 J. Am. Chem. Soc. 129 1478

Ishizaki T and Sakamoto M 2011 Langmuir 272375

Lai Y-K, Gao X-F, Zhuang H-F, Huang J-Y, Lin C-J and Jiang L 2009 Adv. Mater. 213799

Li X-G and Shen J 2011 Chem. Commun. 4710761

Li S-H, Zhang S-B and Wang X-H 2008 Langmuir 245585

Liu M-J, Zheng Y-M, Zhai J and Jiang L 2010 Acc. Chem. Res. 43 368

Liu K-S, Du J-X, Wu J-T and Jiang L 2012 Nanoscale 4768

Manca M, Cannavale A, Marco L-D, Aricò A-S, Cingolani R and Gigli G 2009 Langmuir 256357

Meng H-F, Wang S-T, Xi J-M, Tang Z-Y and Jiang L 2008 J. Phys. Chem. $\mathbf{C 1 1 2} 11454$

Ogihara H, Xie J, Okagaki J and Saji T 2012 Langmuir 28 4605

Shen P, Uesawa N, Inasawa S and Yamaguchi Y 2010 Langmuir 26 13522

Sheng X-I and Zhang J-H 2009 Langmuir 256916

Su B, Li M, Shi Z-Y and Lu Q-H 2009 Langmuir 253640

Tsai H-J and Lee Y-L 2007 Langmuir 2312687

Tuberquia J-C, Nizamidin N, Harl R-R, Albert J, Hunter J, Rogers B-R and Jennings G-K 2010 J. Am. Chem. Soc. 1325725

Wan Y, Min Y-L and Yu S-H 2008 Langmuir 245024

Wang J-X, Zhang Y-Z, Wang S-T, Song Y-I and Jiang L 2011 Acc. Chem. Res. $\mathbf{4 4} 405$

Wenzel R N 1936 Ind. Eng. Chem. 28988

Xiu Y-H, Zhang S, Yelundur V, Rohatgi A, Hess D-W and Wong C-P 2008 Langmuir 2410421 
Xu W-J, Song J-I, Sun J, Lu Y and Yu Z-Y 2011 ACS Appl. Mater. Interf. 34404

Yang L-L, Bai S, Zhu D-S, Yang Z-H, Zhang M-F, Zhang Z-F, Chen E-Q and Cao W-X 2007 J. Phys. Chem. C111 431

Yildirim A, Budunoglu H, Daglar B, Deniz H and Bayindir M 2011 ACS Appl. Mater. Interf. 31804
Zeng H-B, Pesika N, Tian Y, Zhao B-X, Chen Y-F, Tirrell M, Turner K-L and Israelachvili J-N 2009 Langmuir 257486

Zhai L, Cebeci F-Ç, Cohen R-E and Rubner M-F 2004 Nano Lett. 41349

Zhang Y-L, Wang J-N, He Y, He Y-Y, Xu B-B, Wei S and Xiao F-S 2011 Langmuir 2712585 